\title{
Celiac Disease Presenting as Hypokalemic Quadriparesis
}

\author{
Uttayan Chakrabarti*, Manisha Bais Thakur, Ashish Goel \\ Department of Medicine, Vardhman Mahavir Medical College \& Safdarjung Hospital, New Delhi, India \\ *Corresponding author: uttayanchakrabarti@gmail.com
}

Received September 15, 2019; Revised November 12, 2019; Accepted December 04, 2019

\begin{abstract}
Celiac disease is an autoimmune disorder induced by dietary gluten, characterized by a chronic inflammatory state of the small intestinal mucosa, resulting in villous atrophy that resolves with gluten free diet. A small number have classical symptoms and the majority present as atypical celiac disease with absent or few GI symptoms. Celiac crisis is a rare life threatening complication of celiac disease which needs prompt recognition and intervention. We report a rare case of Celiac disease which presented as Celiac crisis with hypokalemic quadriparesis.
\end{abstract}

Keywords: celiac crisis, hypokalemic paralysis

Cite This Article: Uttayan Chakrabarti, Manisha Bais Thakur, and Ashish Goel, "Celiac Disease Presenting as Hypokalemic Quadriparesis.” International Journal of Celiac Disease, vol. 7, no. 3 (2019): 90-91. doi: 10.12691/ijcd-7-3-5.

\section{Introduction}

Hypokalemic paralysis should be considered in any patient presenting with a sudden onset, areflexic, pure motor weakness involving one or more limbs, without alteration in the level of consciousness or sphincter function, and laboratory evidence of hypokalaemia. Any disease entity that results in renal or extrarenal potassium loss is capable of producing hypokalaemic paralysis. It has been found to be associated with Celiac disease, an autoimmune disorder induced by dietary gluten, characterized by a chronic inflammatory state of the small intestinal mucosa, resulting in villous atrophy that resolves with gluten free diet. Here we report a rare case of Celiac disease which presented in Celiac crisis with hypokalemic quadriparesis.

\section{Case Report}

13 year old male with history of 2-3 episodes of painless watery, non-greasy, non-blood mixed loose stools, on and off from 2 years of age presented with 7-8 episodes of loose stools since last 7 days, weakness in all four limbs since 4 days without any history of fever, vomiting, lower limb swelling, bone pains, night blindness, rashes over the body or bleeding from orifices. The weakness started from the right lower limb involving the left lower limb within 24 hours and both upper limbs in next 72 hours without any bladder bowel or cranial nerve involvement. There were no family history, any similar episodes in the past, early morning weakness or precipitation by exercise or carbohydrate rich diet.

On examination, patient was alert, conscious, had a stunted growth, Height-120cm (below 5th percentile),
Weight-23kg (50\% of standard weight for age), BMI-16kg/m², Pallor+, Pulse-108/min, BP-96/58 mm Hg, Mild dehydration+. CNS examination revealed decreased tone in all 4 limbs, Power of 2/5 in B/L lower limbs and $3 / 5$ in $\mathrm{B} / \mathrm{L}$ upper limbs across all joints, with a normal sensory examination. Plantars were bilateral flexor and all deep tendon reflexes were absent. Rest of the systemic examinations were within normal limits.

Investigations revealed Hb-8.8g/dl, MCV-63fl, Hct-27.6\%, MCH-19, MCHC-30\%, TLC-9900/ $\mu \mathrm{L}, \mathrm{Plt}-1,78,000 / \mu \mathrm{L}$, RBS-112mg/dl, Na/K-139/2.2meq/L, U/Cr-15/0.3mg/dl, Total bilirubin-0.8mg/dl, AST/ALT/ALP-527/240/102U/L, Serum total protein-5.9g/dl, Albumin-2.6g/dl, Ca/PO48.1/3 mg/dl. ABG- metabolic acidosis (pH-7.289, Pco2-43.7, HCO3-20.2, So2-96.2), TFT-WNL, HbA1c4.7, Viral markers-NR, Stool R/E, M/E-NAD, ECG- ST depression and $\mathrm{T}$ wave inversion in V1-V4, CXR, USG W/A-NAD. Urine examn: K-3meq/l(11-80), Na-75meq/L (25-301), Cl-89meq/L(27-371), Urine osmolality-196 mosm/Kg. Urine anion gap (Urine $\mathrm{K}+$ Urine $\mathrm{Na}-$ Urine $\mathrm{Cl}$ ) was negative. TTKG (Transtubular potassium gradient) $<2$, which ruled out renal tubular acidosis and proved that the hypokalemia with metabolic acidosis is due to GI cause.

To look for the cause an Upper GI endoscopy was done which revealed absence and scalloping of duodenal folds (Figure 1), biopsy suggestive of Celiac disease, Marsh Oberhuber grading 3b (Figure 2), Anti TTG was markedly raised as well-148 U/ml( $\mathrm{N}<20 \mathrm{U} / \mathrm{ml})$. Patient was given IV fluids and potassium supplementation with complete motor recovery, appearance of deep tendon reflexes and normalisation of potassium levels in 72 hours. Patient was consequently initiated on gluten free diet and iron supplements on discharge and after one month follow up his loose stools had completely subsided, had gained $4 \mathrm{~kg}$ weight \& $3 \mathrm{~cm}$ height with normalisation of lab parameters. (Figure 3) 

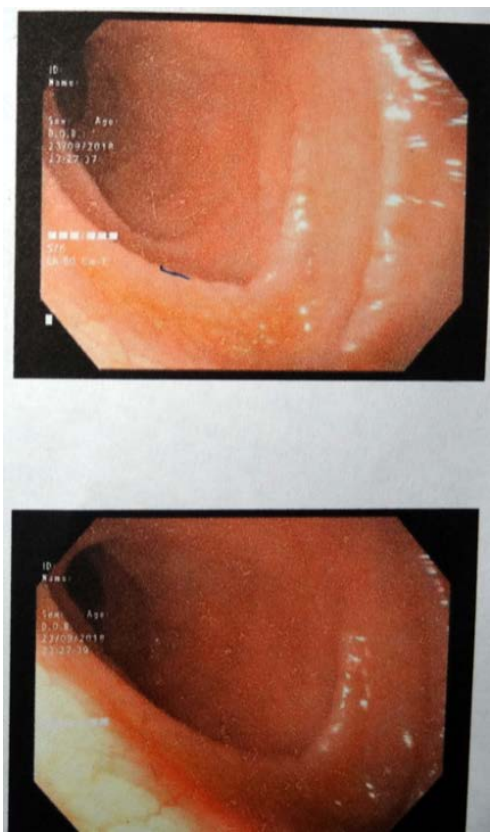

Figure 1. UGIE showing absence and scalloping of duodenal folds

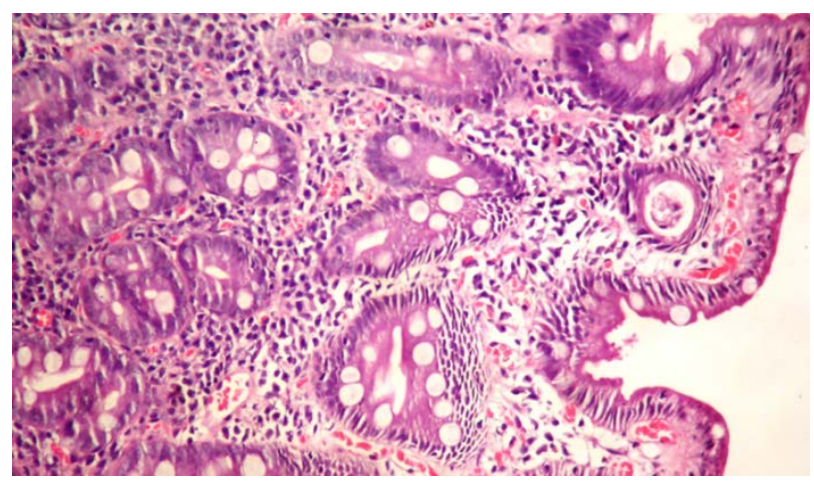

Figure 2. Duodenal biopsy showing severe villous atrophy,crypt hyperplasia,marked chronic inflammation in the lamina propria

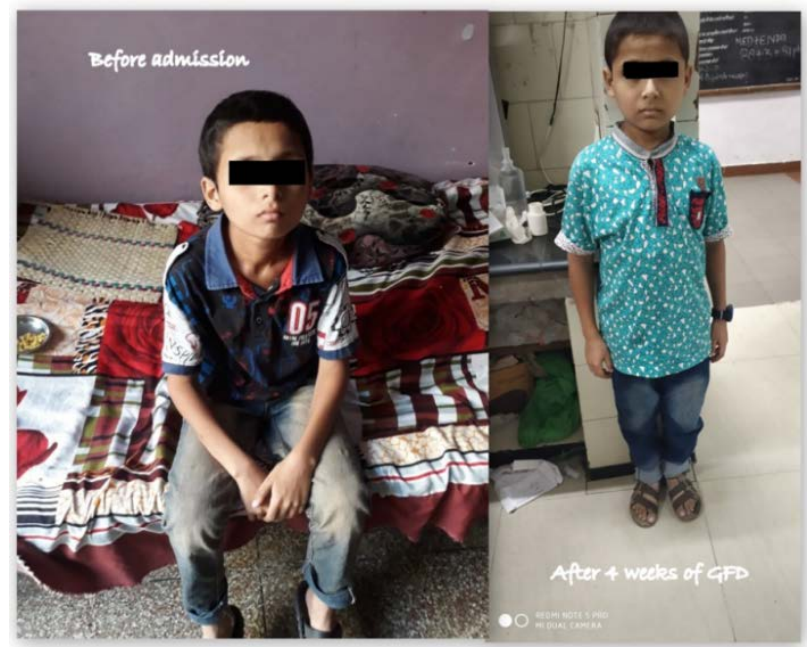

Figure 3. Showing response of the patient to gluten free diet

\section{Discussion}

Anemia is a frequent finding in Celiac, often being the presenting feature with iron deficiency anaemia being reported in upto $46 \%$ of subclinical cases which develops from impaired absorption. Treatment is primarily a gluten free diet and iron supplementation until the iron stores have been restored and the process can take a year for the hemoglobin to normalize and 2 years for the iron stores to replete [1].

Celiac hepatitis is the most common hepatobiliary disorder associated with Celiac, where there is mild to moderate elevation of AST \&/or ALT with normal bilirubin, ALP, GGT. Autoanti bodies other than that of Celiac disease are not found and liver biopsy is of no value. The normalization of aminotransferases with the removal of gluten from the diet suggests a causal relationship between gluten intake/intestinal damage and liver injury [2].

Celiac crisis is defined as an acute onset or rapid progression of GI symptoms attributable to celiac requiring hospitalization and/or parenteral nutrition along with at least 2 of the following-(a) Signs of severe dehydration including hemodynamic instability and/or orthostatic changes, (b) Neurologic dysfunction, (c) Renal dysfunction: creatinine $>2 \mathrm{mg} / \mathrm{dl}$, (d) Metabolic acidosis: $\mathrm{pH}<7.35$, (e) Hypoproteinemia (Albumin $<3 \mathrm{~g} / \mathrm{dl}$ ), (f) Abnormal electrolytes including hyper/hyponatremia, hypocalcemia, hypokalemia or hypomagnesemia, (g) Weight loss $>4.5 \mathrm{~kg}$. [3] Our patient had an exacerbation of loose stools which was associated with metabolic acidosis, hypoproteinemia and hypokalemia and therefore fulfilled the criteria of celiac crisis.

Various precipitating factors identified for crisis are severe malnutrition, infections, hypoproteinemia, and poor compliance to gluten free diet, bacterial overgrowth in setting of altered motility in celiac disease and anticholinergic drugs [4]. In our case the factors being hypoproteinemia and the patient being never on a gluten free diet. Prompt recognition of the condition prevented the patient from succumbing to the life threatening celiac crisis.

\section{References}

[1] Hoffbrand AV. Anaemia in adult coeliac disease.Clin Gastroenterol. 1974; 3: 71-89.

[2] Bardella MT, Fraquelli M, Quatrini M, Molteni N, Bianchi P, Conte D. Prevalence of hypertransaminasaemia in adult celiac patients and effects of gluten-free diet. Hepatology. 1995; 22: 833-6.

[3] Jamma S,Tapia AR , Kelly CP, Murray J, Sheth S, Schuppan D, et al. Celiac crisis is a rare but serious complication of celiac disease in adults. Clin Gastroenterol Hepatol. 2010 ; 8(7): 587-590.

[4] Venkateswarlu K, Prasad RG, Malik AK, Nagi B, Mehta S. Coeliac disease. Indian Pediatr 1984; 21, 897-900. 